\title{
Research on PSD Replication for Multiaxial Hydraulic Vibration Test System Based on FXLMS Algorithms
}

\author{
Yunbin Cheng $\mathbb{D}^{1,2}$ Bin $\mathrm{Li}\left(\mathbb{D}^{1},{ }^{1}\right.$ Jun Wang, ${ }^{2}$ and Heliang $\mathrm{Li}^{2}$ \\ ${ }^{1}$ School of Mechatronic Engineering and Automation, Shanghai University, Shanghai 200444, China \\ ${ }^{2}$ Shanghai Institute of Quality Inspection and Technical Research, Shanghai 200233, China \\ Correspondence should be addressed to Bin Li; sulibin@shu.edu.cn
}

Received 29 December 2020; Revised 5 May 2021; Accepted 17 August 2021; Published 6 September 2021

Academic Editor: Jiaxi Zhou

Copyright ( 92021 Yunbin Cheng et al. This is an open access article distributed under the Creative Commons Attribution License, which permits unrestricted use, distribution, and reproduction in any medium, provided the original work is properly cited.

The multiaxial electrohydraulic vibration test system can not only simulate the multiexcitation on multidimensional vibration environment but also meet the vibration test requirements of high thrust, large displacement, low frequency, etc. In order to eliminate the effects of nonlinear factor and system noise and achieve a more accurate control result, a control algorithm based on the frequency-domain filtered-x least mean square adaptive algorithm (FXLMS) is proposed to achieve the power spectral density (PSD) replication. The main idea is to use the frequency-domain FXLMS algorithm to adjust the controller adaptively corresponding to the transfer function uncertainty and changes of the plant, which are typically caused by time-varying parameters in electrohydraulic actuators and system noise. The details and implantation steps of the proposed algorithm are analysed for the single-input single-output electrohydraulic vibration test system. The proposed algorithm and control strategy are then extended to the multiaxial electrohydraulic vibration test system. Eventually, some experimental targets for performing the PSD replication test on a two-exciter system are carried out, in which the results show that the proposed algorithm is valid and meets the test standards..

\section{Introduction}

Dynamics testing is important for detecting the mechanical properties of structures and products [1]. The electrohydraulic shaking table (EHST) is one of the most important vibration test system and widely used in the fields of architectural engineering [2], civil engineering [3], earthquake resistance testing $[4,5]$, mechanical fatigue [6], structural testing [1], and so on. There are two categories of shaking tables targeting different control objects, namely, displacement-controlled and acceleration-controlled hydraulic shaking tables. The majority of the early EHST belongs to the former due to a simple control scheme, which has the shortcomings of a complex operation. The accelerationcontrolled hydraulic shaking tables are now widely adopted [3].

The control of the actuators of EHSTs, including both magnitude and phase control, is vital to replicate the reference signals with small deviations. The goal is challengeable due to the complex and nonlinear characteristics of the shaking table $[1,3,7,8]$. Furthermore, when conducting multi-input multioutput (MIMO) random vibration environment tests, response spectral lines of testing systems may exceed their tolerances. The reason for this phenomenon is the high-level noise in the input forces, which are induced by the inverse of the ill-conditioned frequency response function matrices, the system uncertainty caused by time-varying parameters, and system noise in electrohydraulic actuators. These spectral lines may even trigger the instability of control algorithms and eventually result in an accidental test shutoff $[9,10]$. There are some algorithms which have been studied, such as PID algorithm and least mean squares (LMS) algorithm, but they suffer from the weakness of small frequency bandwidth and poor tracking accuracy. The Filtered-x Least Mean Square (FXLMS) algorithm was derived by Widrow in 1981 and has been extensively used in applications, such as active noise control (ANC) and active vibration control (AVC). The basic 
idea of FXLMS is to adjust the drive signal to achieve the control target adaptively. The frequency-domain FXLMS algorithm is a transform of normal FXLMS algorithms that process sample blocks rather than point by point. Since the object is unknown, the adaptive system is required to identify or adjust the parameters of the controller to obtain the inverse of the controlled object. Taking the inverse of the transfer function of the controlled object as a series controller, the adaptive inverse control (AIC) is used for the open-loop control of the dynamic characteristics of the system, which can avoid the instability of the system arising from feedback, and to independently separate the control of the dynamic characteristics of the system and the control of the disturbance of the controlled object.

Several PSD replication algorithms based on LMS algorithms have been developed in recent decades. Salehzadeh-Nobari et al. developed the frequency-domain FXLMS from Ling Dynamic System (LDS) and applied it to the shock vibration test of the vibration table [11]. Karshenas et al. proposed an impact test algorithm based on the LMS control algorithm, which incorporates the standard FXLMS control structure with the frequency-domain adaptive filter [12]. The shock waveform was successfully reproduced in their experiment. Yang et al. proposed an adaptive inverse control of random vibrations based on the FXLMS algorithm, and the frequency-domain LMS algorithm was adopted to refine the inverse characteristics of the frequency response functions (FRFs) [13]. Shen et al. [14] proposed a control strategy combining the AIC and inverse frequency response function (IFRF) and applied it to the EHST. The performance of system frequency bandwidth and asymptotic reference tracking was significantly improved in their experiment compared with traditional PID and IFRF controls. Later, an effective algorithm that combines feed-forward inverse control with internal model control (IMC) and realtime feedback control was proposed to further increase the control accuracy for EHST [15]. The present algorithms are still limited by high requirements on computational speed and efficiency of controllers, which leaves a gap for the real engineering tests. In this work, we propose an improved AIC based on the FXLMS algorithm and test it on a multiaxial hydraulic vibration test system. The algorithm is tested on a two-exciter system, and the experimental results suggest that it can significantly reduce the nonlinearity and noise effect.

\section{Theory and Method}

2.1. The Principle of the Frequency-Domain FXLMS Algorithm. The block diagram of frequency-domain FXLMS algorithm [16] is shown in Figure 1. $Z(f)$ is the controller, which is connected with the plant in series. $H(f)$ is the transfer function of the plant, and $\widehat{H}(f)$ is an estimation of $H(f)$. $M(f)$ is a reference model that plays a role as performance index required by the control system. $R(f)$ is the reference signal for the response $Y(f)$ of the plant. $D(f)$ is the driver signal, and $E(f)$ is the error signal between the response and the target. The implementation processes of the frequencydomain FXLMS algorithm can be summarized as follows:

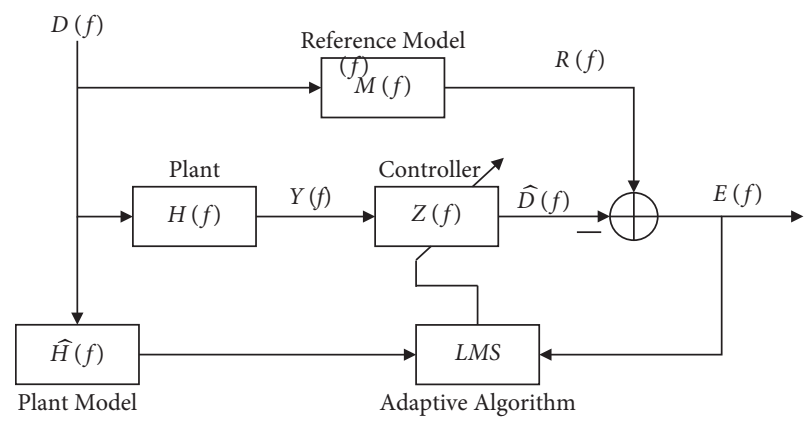

FIGURE 1: Block diagram of frequency-domain FXLMS algorithm.

(1) Calculate the FFT coefficient matrixes of the reference signal block $\mathbf{r}(k)$, drive signal block $d(k)$, and response signal block $\mathbf{y}(k)$ at the $k$ th iteration:

$$
\begin{aligned}
& \mathbf{R}_{k}(f)=d \operatorname{FFT}[\mathbf{r}(k)]^{T}, \\
& \mathbf{D}_{k}(f)=\operatorname{FFT}[\mathbf{d}(k)]^{T}, \\
& \mathbf{Y}_{k}(f)=\operatorname{diag}\left\{\operatorname{FFT}[\mathbf{y}(k)]^{T}\right\},
\end{aligned}
$$

where $\mathbf{r}(k), \mathbf{d}(k)$, and $\mathbf{y}(k)$ are expressed as $\mathbf{r}(k)=$ $[r(k N), r(k N+1), \ldots, r(k N+N-1)], \quad \mathbf{d}(k)=$ $[d(k N), d(k N+1), \ldots, d(k N+N-1)]$, and $\mathbf{y}(k)=$ $[y(k N), y(k N+1), \ldots, \quad y(k N+N-1)], \quad$ respectively. $N$ is the block size.

(2) Update the controller $Z(f)$. The error signal $E(f)$ can be obtained easily from Figure 1 as

$$
\begin{aligned}
E_{k}(f) & =R_{k}(f)-Y_{k}(f) \\
& =D_{k}(f)\left[M(f)-H(f) Z_{k}(f)\right],
\end{aligned}
$$

where $z(k)=\left\{z_{i}(k)\right\},(i=0,1, \ldots, N-1)$, is the FIR model of the controller in time-domain and $Z(f)=\operatorname{FFT}(z(k))^{T}$ is the FFT of the controller. When the adaptive algorithm converges, the error signal achieves the minimum value, which means $E(f) \approx 0$. Then, the controller $Z(f)$ can be drawn from equation (2) as follows:

$$
Z(f)=\frac{M(f)}{H(f)}
$$

In this paper, $M(f)$ has been chosen as $M(f)=1$. Then, the transfer function of the controller $Z(f)=$ $H^{-1}(f)$ is the inverse transfer function (impedance function) of the plant. According to the steepest descent algorithm, the coefficients of the controller can be updated as

$$
Z_{k+1}(f)=Z_{k}(f)+\mu D_{k}^{*}(f) \hat{H}(f) E_{k}(f),
$$

where $\mu$ is the step size and affects the convergence rate and steady state error. The value of $\mu$ should be chosen carefully to balance the convergence rate and steady state error. The convergence condition of the frequency-domain FXLMS algorithm is 


$$
0<\mu<\frac{2}{\lambda_{\max , k}},
$$

where $\lambda_{\max , k}$ is the maximum eigenvalue of the autocorrelation matrix of the response $y(k)$ in the $k$ th iteration.

(3) Calculate the drive signal $D_{k+1}(f)$ by using the following equation:

$$
D_{k+1}(f)=Y_{k}(f) Z_{k+1}(f) .
$$

2.2. PSD Replicate Algorithm for SISO Test System. A novel algorithm that combines the frequency-domain FXLMS algorithm and the basic closed-loop control diagram is proposed. It facilitates more flexibility and can be extended to multiaxial test system easily. The control flowchart of the proposed algorithm based on the single-input single-output (SISO) system is shown in Figure 2.

$G_{r r}(f), G_{d d}(f), G_{y y}(f)$, and $G_{e e}(f)$ are the self-power spectral densities of the target, drive signal, response signal, and control error, respectively. $\beta$ is the correction factor that controls the correction rate of the drive signal. $\widehat{Z}(f)=\widehat{H}^{-1}(f)$ is the inverse model of $\widehat{H}(f)$. As the system noise and time-varying factors in plant cannot be avoided, the transfer function $H(f)$ has to be estimated online and the drive signal $\mathbf{d}(k)$ has to be corrected iteratively. The implementation process of the proposed PSD replication algorithm is as follows:

(1) Estimate the initial transfer function $\widehat{H}_{0}(f)$ and the initial impedance function $\widehat{Z}_{0}(f)$ in the pretest:

$$
\begin{aligned}
& \widehat{H}_{0}(f)=\frac{G_{d y}(f)}{G_{d d}(f)}, \\
& \widehat{Z}_{0}(f)=\widehat{H}_{0}^{-1}(f),
\end{aligned}
$$

where $G_{d y}(f)$ is the cross-power spectral density of drive signal $\mathbf{d}(k)$ and response signal $\mathbf{y}(k)$. Equation (7) is called $\mathrm{H}_{2}$ method.

(2) For the $k$ th iteration, update the controller $\widehat{Z}_{k+1}(f)$ by the frequency-domain FXLMS algorithm descripted in Section 2 as follows:

$$
\begin{aligned}
E_{k}(f) & =Y_{k}(f) Z_{k}(f)-D_{k}(f), \\
\widehat{Z}_{k+1}(f) & =\widehat{Z}_{k}(f)+\mu D_{k}^{*}(f) H_{k}(f) E_{k}(f) .
\end{aligned}
$$

(3) Then, the corrected drive signal $G_{d}^{\prime}(f)$ can be drawn as follows:

$$
G_{d d}^{\prime}(f)=G_{d d}(f)+\beta \widehat{Z}^{*}(f) G_{e e}(f) \widehat{Z}(f),
$$

where $G_{d d}(f)$ and $G_{e e}(f)$ are

$$
\begin{aligned}
G_{d d}(f) & =Z^{*}(f) G_{r r}(f) Z(f), \\
G_{e e}(f) & =G_{y y}(f)-G_{r r}(f) .
\end{aligned}
$$

After obtaining the corrected drive signal $G_{d}{ }_{d}^{\prime}(f)$, it can be converted to time-domain signal $\mathbf{d}(k)$ and used to drive the EHSTs.

2.3. PSD Replicate Algorithm for MIMO Test System. A multiinput and multioutput (MIMO) PSD algorithm is developed based on the SISO algorithm introduced in Section 2.2. The block diagram of the proposed PSD replication algorithm for MIMO system is shown in Figure 3.

The white noise matrix $[W]$ is specifically used to improve the accuracy of estimation and eliminate the static friction of the hydraulic system. $\left[G_{r r}\right]$ is the PSD matrix of the control target. $[L]$ is the PSD matrix of the drive signal after decoupling. The transmission characteristics of the multiaxial hydraulic vibration test system can be expressed as

$$
\left\{\begin{array}{c}
y_{1}(f) \\
y_{2}(f) \\
\vdots \\
y_{m}(f)
\end{array}\right\}=\left[\begin{array}{cccc}
h_{11}(f) & h_{12}(f) & \cdots & h_{1 m}(f) \\
h_{21}(f) & h_{22}(f) & \cdots & h_{2 m}(f) \\
\vdots & \vdots & \ddots & \vdots \\
h_{n 1}(f) & h_{n 2}(f) & \vdots & h_{n m}(f)
\end{array}\right]\left\{\begin{array}{c}
d_{1}(f) \\
d_{2}(f) \\
\vdots \\
d_{m}(f)
\end{array}\right\} .
$$

That is,

$$
Y(f)=[H(f)] D(f),
$$

where $H(f)$ is the transfer function matrix, $D(f)$ is the drive signal vector, and $Y(f)$ is the response signal vector.

Again, the main idea of the proposed algorithm is to update the system impedance matrix $[Z]$ by the frequencydomain FXLMS algorithm. The implementation process of the proposed PSD replication algorithm for MIMO system is as follows:

(1) Estimate the initial transfer function matrix $\left[\widehat{H}_{0}(f)\right]$ and the initial impedance function matrix $\left[\widehat{Z}_{0}(f)\right]$ in the pretest. $H_{v}$ estimation algorithm is adopted for MIMO system. From Figure 3, the error signal vector $E(f)$ can be obtained by

$$
E(f)=Y(f)-[H(f)] D(f) .
$$

Take the expectation of $E(f)$ as

$$
E\left[E(f) E^{H}(f)\right]=E\left\{[Y(f)-[H(f)] D(f)][Y(f)-[H(f)] D(f)]^{H}\right\}
$$




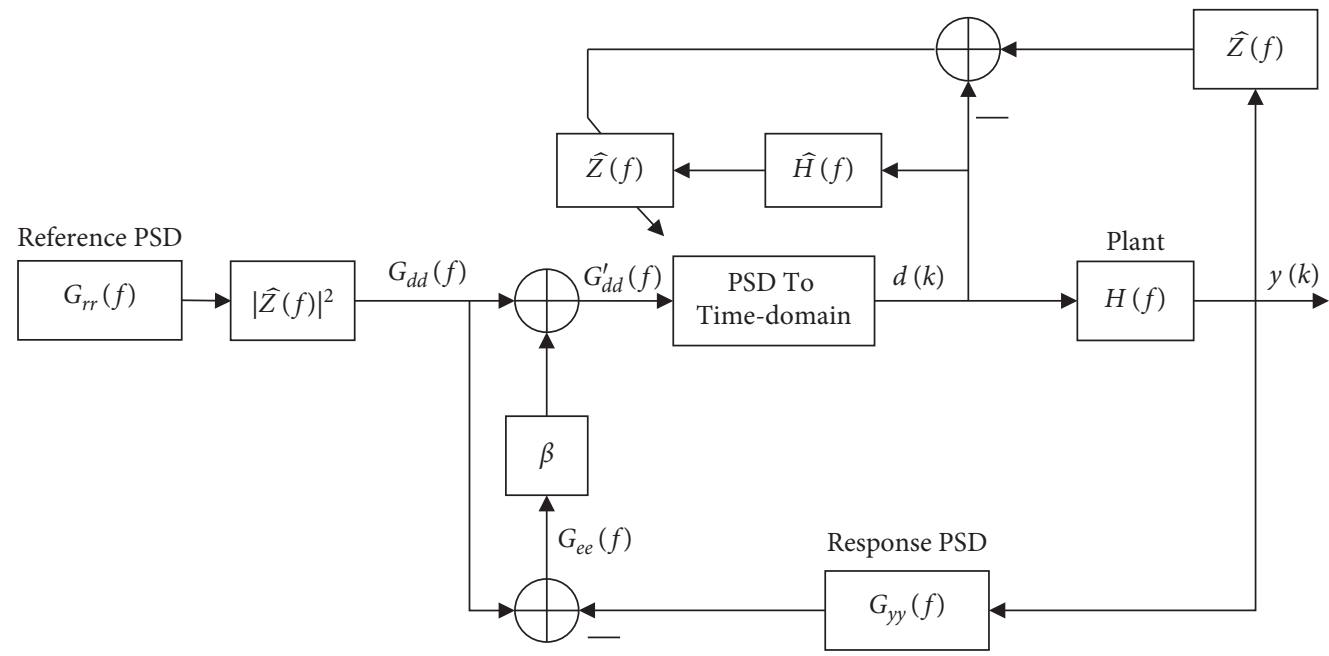

FIGURE 2: The block diagram of the proposed PSD replication algorithm in SISO system.

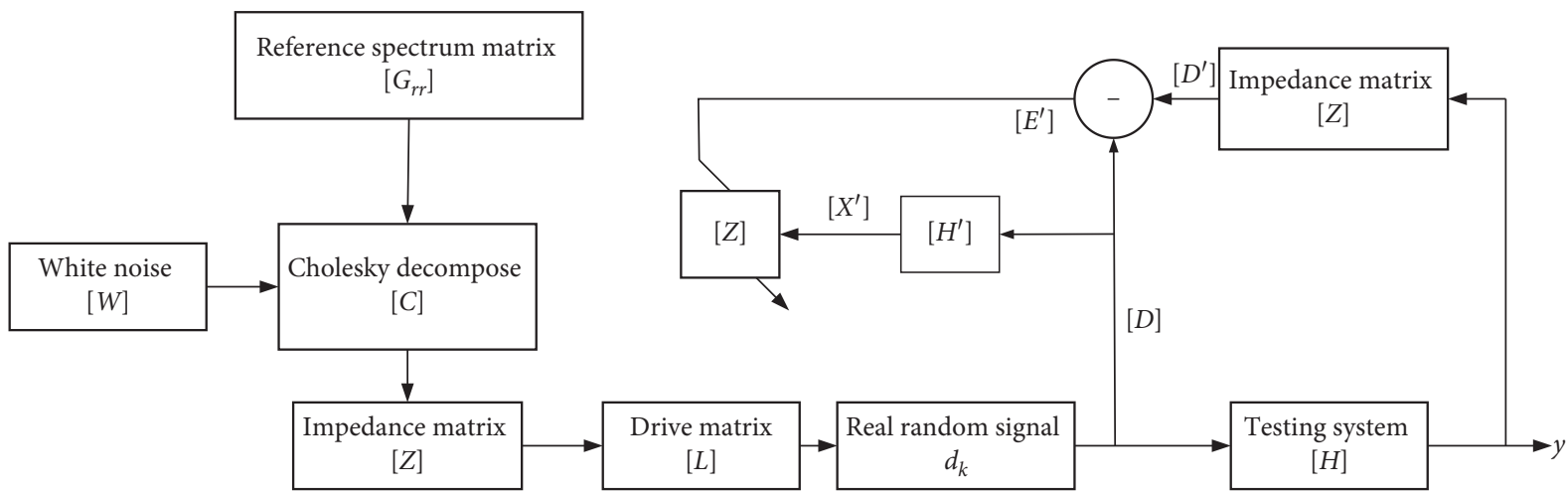

FIGURE 3: The block diagram of the proposed PSD replication algorithm for MIMO test system. [.] is used to represent the matrix form.

Let $\left[G_{e e}\right]=E\left[E(f) E^{H}(f)\right]$ and rewrite it as

$$
\left[G_{e e}\right]=\left[I H_{v}\right]\left[\begin{array}{cc}
G_{y y} & -G_{y d} \\
-G_{d y} & G_{d d}
\end{array}\right]\left[\begin{array}{c}
I \\
H_{v}
\end{array}\right] .
$$

According to the principle of $H_{v}$ algorithm, the trace of $\left[G_{e e}\right]$ should be minimized. The initial transfer function matrix $\left[\widehat{H}_{0}(f)\right]$ can be obtained as

$$
\left[H_{0}\right]=\frac{G_{y y}-G_{d d}+\sqrt{\left(G_{y y}-G_{d d}\right)^{2}+4\left|G_{d y}\right|^{2}}}{2 G_{d y}} .
$$

In order to improve the accuracy, the estimated matrix $\left[\hat{H}_{0}(f)\right]$ is calculated several times and averaged by

$\left[H_{k}(f)\right]= \begin{cases}{\left[H_{0}(f)\right],} & k=0, \\ (1-\beta)\left[H_{k-1}(f)\right]+\beta\left[H_{k}(f)\right], & i=1,2, \ldots, N,\end{cases}$

where $k$ is the iteration time and $\beta$ is the step size. Then, the impedance function matrix $\left[\widehat{Z}_{0}(f)\right]$ can be easily obtained as

$$
\left[\widehat{Z}_{k}(f)\right]=\left[\widehat{H}_{k}(f)\right]^{-1} .
$$

(2) For the $j$ th iteration, update the controller matrix $\left[\widehat{Z}_{k+1}\right]$ by the frequency-domain FXLMS algorithm as follows:

$$
[Z]_{j+1}=[Z]_{j}+2 \mu[E \prime]_{j}[X]_{j}^{H},
$$

where $\left[X^{\prime}\right]=\left[H^{\prime}\right]_{j}[D]_{j}$ and $[E]_{j}=[D]_{j}-[Z]_{j}$ $[H]_{j}[D]_{j}$. Simplify equation (20) to

$$
\begin{aligned}
{[Z]_{j+1} } & =[Z]_{j}+2 \mu\left([D]_{j}-[Z]_{j}[H]_{j}[D]_{j}\right)\left(\left[H^{\prime}\right]_{j}[D]_{j}\right)^{H} \\
& =[Z]_{j}+2 \mu\left(I-[Z]_{j}[H]_{j}\right)\left[G_{d d}\right]_{j}[H]_{j}^{H} .
\end{aligned}
$$

Furthermore, equation (21) can be transferred into PSD form as

$$
\begin{aligned}
{[Z]_{j+1} } & =[Z]_{j}+2 \mu\left(\left[G_{d d}\right]_{j}-\left[G_{d, d_{1}}\right]\right)\left([H \prime]_{j}\left[G_{d d}\right]_{j}\right)^{H} \\
& =[Z]_{j}+2 \mu[Z]_{j}\left(\left[G_{r r}\right]_{j}-\left[G_{y y}\right]_{j}\right)[Z]_{j}^{H}\left([H \prime]_{j}\left[G_{d d}\right]_{j}\right)^{H},
\end{aligned}
$$


where

$$
[E]_{j}=[D]_{j}-[Z]_{j}[\widehat{H}]_{j}[D]_{j}
$$

Further simplify equation (22) to

$$
\begin{aligned}
{[Z]_{j+1} } & =[Z]_{j}+2 \mu\left([D]_{j}-[Z]_{j}[H]_{j}[D]_{j}\right)\left([H \prime]_{j}[D]_{j}\right)^{H} \\
& =[Z]_{j}+2 \mu\left(I-[Z]_{j}[H]_{j}\right)\left[G_{d d}\right]_{j}[H]_{j}^{H} .
\end{aligned}
$$

(3) Calculate the drive signal $\left[G_{d d}\right]_{j+1}$ :

$$
\left[G_{d d}\right]_{j+1}=\left[G_{d d}\right]_{j}+\alpha[Z]_{j+1}\left[G_{e e}\right][Z]_{j+1}^{H} \text {. }
$$

After obtaining the corrected drive signal $\left[G_{d d}\right]_{j+1}$, it can be converted to time-domain signal $[\mathbf{d}]_{j+1}$ and used to drive the EHST.

\section{Results and Discussions}

3.1. Control Test of Biaxial Hydraulic Vibration Test System. In order to further verify the controlling effects of frequencydomain FXLMS algorithm on the hydraulic vibration test system, the dual and parallel hydraulic pressure vibration test system (Figure 4) developed by Zhejiang University, Shanghai Institute of Quality Inspection and Technical Research, and Hangzhou Econ Science and Technology Company is used as a test object to carry out pilot studies. The core controller of this system adopts the Premax VT series vibration controller of Hangzhou Econ Technology Co., Ltd. Other key devices include hydraulic vibration test bench, pump station, and oil separator. The working parameters of the hydraulic vibration test are shown in Table 1.

3.2. Self-Closed Loop Test. The power spectral reproduction self-closed loop test process is shown in Figure 5. Output 2 channel and Input 2 channel are selected for test verification. In the self-closed loop test, two reference spectral curves of random power spectral density were selected. The experimental bandwidth was $5 \mathrm{~Hz} \sim 100 \mathrm{~Hz}$, and the effective value of the control signal was $0.1 \mathrm{~g}$. The correlation coefficient between signals was 0.3 .

The results of the power spectral reproduction loop test are shown in Figure 6, where Figures 6(a) and 6(b) show the control spectral of Channels 1 and 2, respectively. Figure 6(c) shows the control signals of Channels 1 and 2. Figure 6(d) shows the control curve of the coherence coefficient between Channels 1 and 2. From the results of the power spectral reproduction closed-loop control test, it can be seen that the power spectral reproduction test based on our improved frequency-domain FXLMS adaptive inverse control algorithm can achieve high accuracy with a closed-loop control. The control spectral accuracy meets the test requirements well.

3.3. Field Test. The field test of power spectral reproduction is shown in Figure 7. Taking into account the coupling characteristics of the vibration test system, a motorcycle is

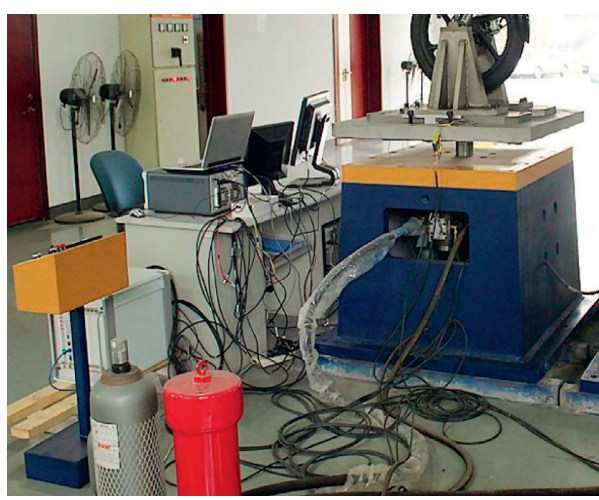

Figure 4: The hydraulic vibration test system.

TABLE 1: The main parameters of hydraulic vibration test system in Figure 4.

\begin{tabular}{lc}
\hline Name & Technical data \\
\hline Table-board quality & $80 \mathrm{~kg}$ \\
Table-board size & $1 \mathrm{~m} \times 1 \mathrm{~m}$ \\
Load quality & $100 \mathrm{~kg}$ \\
Exciter working area $A_{p}$ & $1.5 \times 10^{-3} \mathrm{~m}^{2}$ \\
Volume of the oil chamber $V$ & $1.67 \times 10^{-4} \mathrm{~m}^{3}$ \\
Oil volume elastic modulus $\beta$ & $7 \times 10^{8} \mathrm{~N} / \mathrm{m}^{2}$ \\
Flow pressure coefficient $K_{c}$ of servo valve & $1.38 \times 10^{-11} \mathrm{~m}^{5} / \mathrm{Ns}$ \\
Exciter working stroke & $0.112 \mathrm{~m}$ \\
The coefficient of pipeline $\eta$ & 1.1 \\
\hline
\end{tabular}

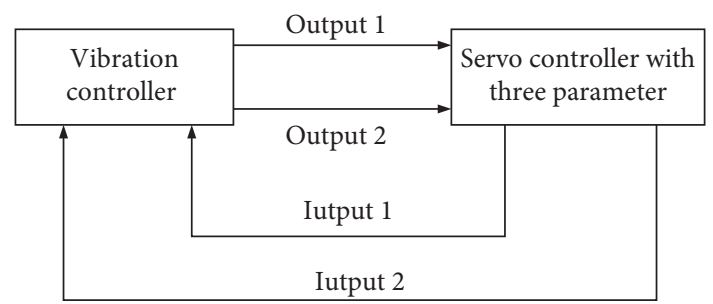

FIgURE 5: The diagram of the dual closed-loop channel control test.

selected as the test object. The dual excitations and dual control modes were adopted. The power spectral designed in the self-closed loop test is used as the control reference spectral.

After four valid iterations, the field test results of the power spectral reproducibility control of the dual and parallel hydraulic pressure vibration test system are shown in Figure 8. Figures 8(a) and 8(b) show the power spectral density curves of control signals, respectively. Figure 8(c) shows the (time domain) control signal of Channels 1 and 2 in the field test. Figure $8(\mathrm{~d})$ shows the control curve of the coherence coefficient between control signals, and Figure 8(e) is the system impedance curve.

From the field test results of the power spectral reproducibility control of the test system, it can be seen that, within the test frequency bandwidth $5 \mathrm{~Hz} \sim 100 \mathrm{~Hz}$, the selfpower spectral density curves of the control signal are basically controlled within the error range of $\pm 1.5 \mathrm{~dB}$ (Figures 8(a) and 8(b)), which meets the test standard well. 

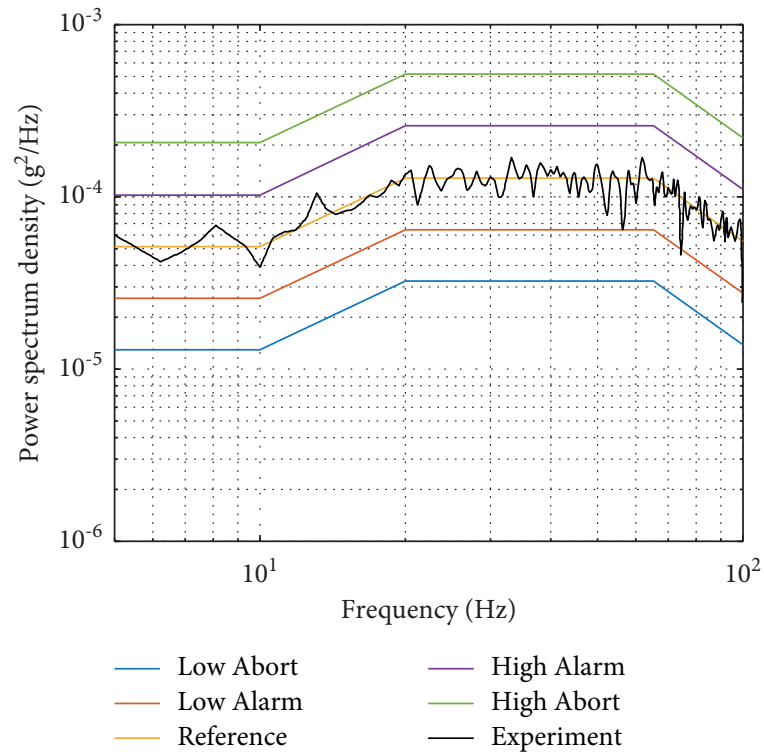

(a)

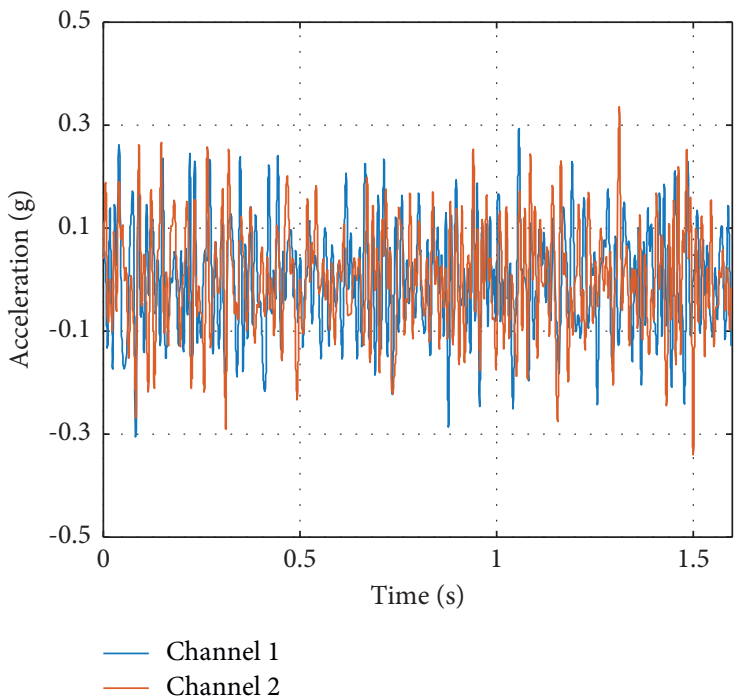

(c)
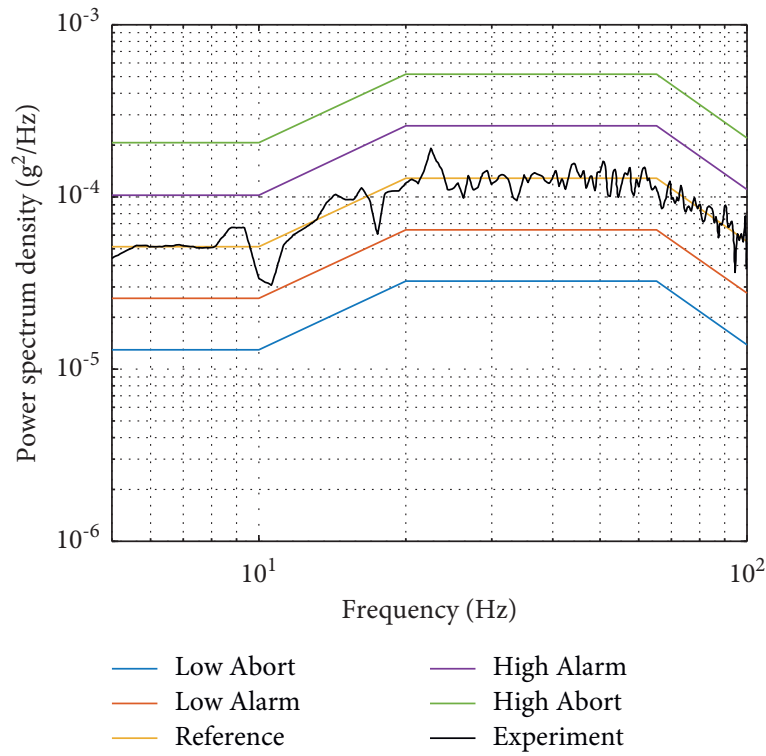

(b)

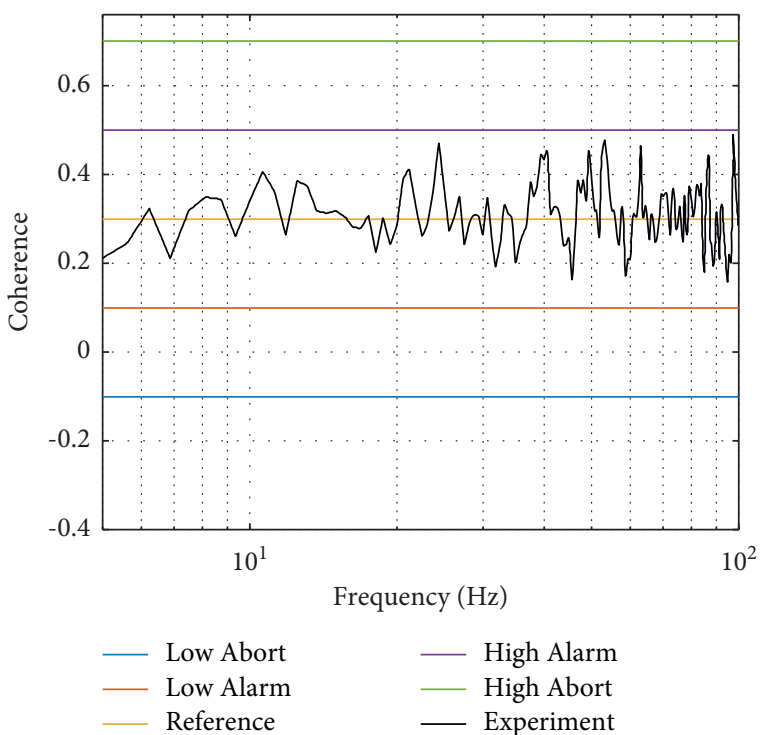

(d)

Figure 6: The results of the dual-channel control test. (a) The control spectral of Channel 1. (b) The control spectral of Channel 2. (c) The two control signals of Channels 1 and 2. (d) The coherence between the two channels.

The coherence coefficient and the impedance curves are shown in Figures 8(d) and 8(e), respectively. It can be that there is an antiresonant frequency around $40 \mathrm{~Hz}$, which makes the coherence coefficient outside the limit. However, the overall control effect is essentially controlled within the range of control errors. The classical control algorithms for MIMO random vibration environment tests have difficulty on the resonant frequency and are sensitive to the system uncertainty [17-19]. The proposed algorithm maintains better stability and robustness. From the field test results, it can be drawn that the proposed algorithm overcomes the antiresonant frequency, system uncertainty, and system noise effectively, while maintaining a good control result that meets the test standards.

\section{Summary}

In this paper, the improved adaptive inverse control algorithm and the power spectral recurrent control algorithm are investigated and verified with the multiaxial vibration test system. By adopting the power spectral reproduction control based on the frequency-domain FXLMS adaptive inverse control algorithm, the system impedance matrix can be adaptively corrected and the closed-loop control of the system drive signal can be completed. The field test on the two-exciter system shows the effectiveness of the algorithm in PSD replication. The algorithm can effectively restrain the influence of nonlinear factors such as interference noise in the system on the power spectral density reproduction 


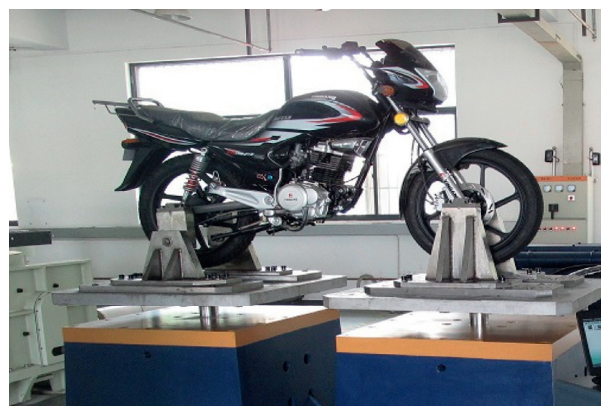

Figure 7: The field test using the two-exciter hydraulic vibration test system.
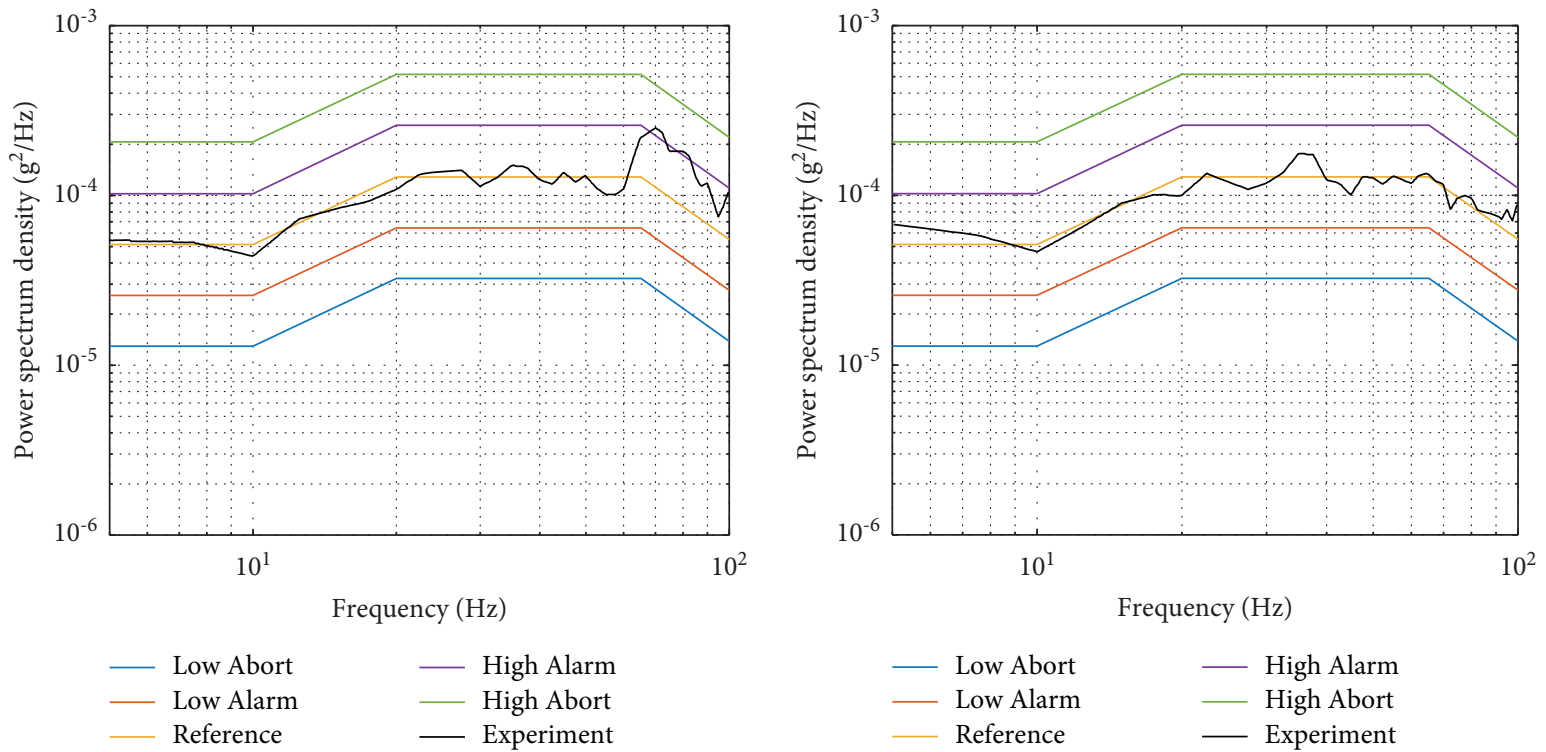

(a)

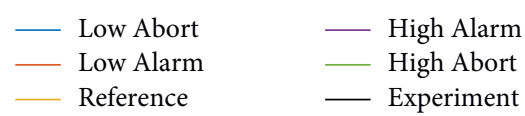

(b)
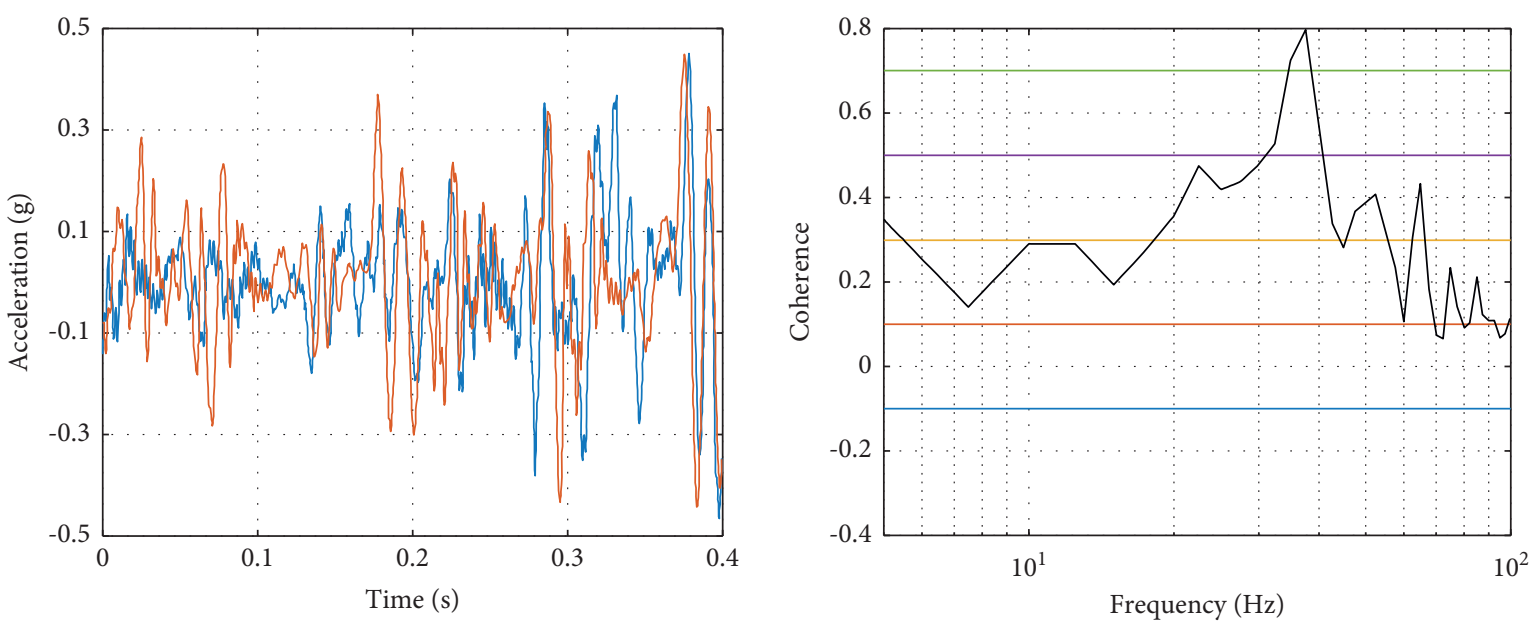

Channel 1

Channel 2

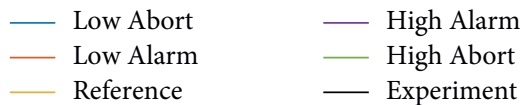

(c)

(d)

FIgURE 8: Continued. 


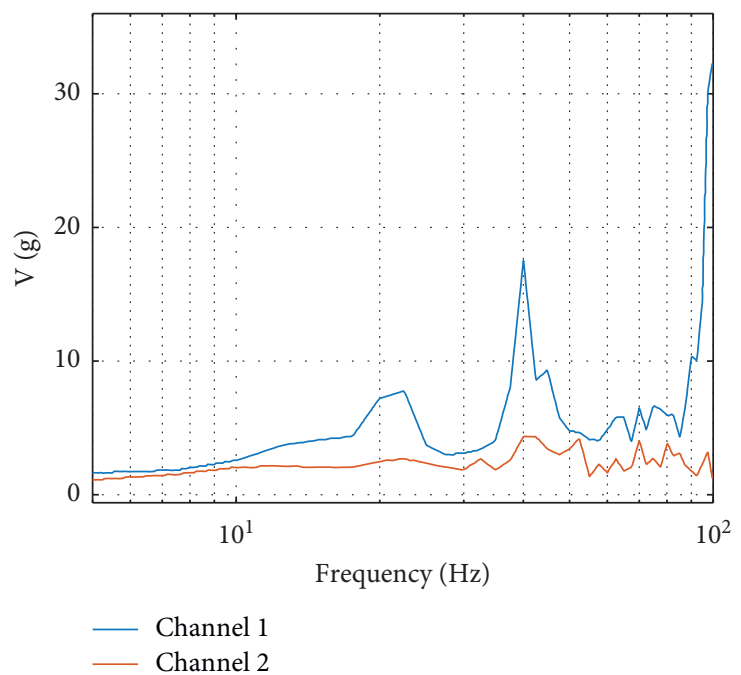

(e)

FIgURE 8: The test results of the field test using the dual-channel hydraulic vibration test system. (a) The control spectral of Channel 1 in the field test. (b) The control spectral of Channel 2 in the field test. (c) The two control signals of channels in the field test. (d) The coherence between the two channels in the field test. (e) The impedance curves of the two channels in the field test.

control of a multiaxial hydraulic vibration test system and thus significantly improve the precision of system control. Although the present algorithm is well investigated, there are still some areas open to further research. The antiresonant frequency has a significant impact on control accuracy. Concerning this, more control strategies should be studied to improve the control accuracy around the antiresonant frequency.

\section{Data Availability}

The data used to support the findings of this study are included within the article.

\section{Conflicts of Interest}

The authors declare no conflicts of interest in preparing this article.

\section{References}

[1] A. R. Plummer, "Control techniques for structural testing: a review," Proceedings of the Institution of Mechanical Engineers Control Engineering, vol. 221, no. 2, pp. 139-169, 2007.

[2] R. T. Severn, "The development of shaking tables-a historical note," Earthquake Engineering \& Structural Dynamics, vol. 40, no. 2, pp. 195-213, 2011.

[3] J. Yao, M. Dietz, R. Xiao, H. Yu, T. Wang, and D. Yue, "An overview of control schemes for hydraulic shaking tables," Journal of Vibration and Control, vol. 22, no. 12, pp. 2807-2823, 2016.

[4] S. A. Neild, D. P. Stoten, D. Drury, and D. J. Wagg, "Control issues relating to real-time substructuring experiments using a shaking table," Earthquake Engineering \& Structural Dynamics, vol. 34, no. 9, pp. 1171-1192, 2005.

[5] X. Ji, K. Kajiwara, T. Nagae, R. Enokida, and M. Nakashima, "A substructure shaking table test for reproduction of earthquake responses of high-rise buildings," Earthquake
Engineering \& Structural Dynamics, vol. 38, no. 12, pp. 1381-1399, 2009.

[6] D. P. McCrum and M. S. Williams, "An overview of seismic hybrid testing of engineering structures," Engineering Structures, vol. 118, pp. 240-261, 2016.

[7] M. K. Thompson, Mil-Std-810g Environmental Engineering Considerations and Laboratory Tests, US Department of Defense, Washington, DC, USA, 2008.

[8] C. A. S. A. T. Corporation, Multi-Dimension Vibration Tests, CASIC, Beijing, China, 2013.

[9] S. Cui, H. Chen, X. He, and W. Zheng, "Control algorithm update for multi-input multi-output random environment test," Mechanical Systems and Signal Processing, vol. 111, pp. 643-662, 2018.

[10] M. A. Underwood, "Adaptive control method for multiexciter sine tests," Google Patents, 1994.

[11] S. Salehzadeh-Nobari, J. Chambers, T. Green, J. Goodfellow, and W. Smith, "Implementation of frequency domain adaptive control in vibration test products," in Proceedings of the 5th International Conference on Factory 2000-The Technology Exploitation Process, Cambridge, UK, April 1997.

[12] A. M. Karshenas, B. W. Williams, and M. W. Dunnigan, "Adaptive inverse control algorithm for shock testing," IEE Proceedings-Control Theory and Applications, vol. 147, no. 3, pp. 267-276, 2000.

[13] Z. Yang, Q. Huang, J. Han, and H. Li, “Adaptive inverse control of random vibration based on the filtered-X lms algorithm," Earthquake Engineering and Engineering Vibration, vol. 9, no. 1, pp. 141-146, 2010.

[14] G. Shen, S.-T. Zheng, Z.-M. Ye, Q.-T. Huang, D.-C. Cong, and J.-W. Han, "Adaptive inverse control of time waveform replication for electrohydraulic shaking table," Journal of Vibration and Control, vol. 17, no. 11, pp. 1611-1633, 2011.

[15] G. Shen, G.-M. Lv, Z.-M. Ye, D.-C. Cong, and J.-J. Han, "Feedforward inverse control for transient waveform replication on electro-hydraulic shaking table," Journal of Vibration and Control, vol. 18, no. 10, pp. 1474-1493, 2012. 
[16] B. Widrow, "Adaptive inverse control," in Adaptive Systems in Control and Signal Processing 1986Elsevier, Amsterdam, Netherland, 1987.

[17] Y. Tian, H. Chen, F. Shen, and M. Bao, "Dual-shaker random vibration decoupling control based on $\mathrm{H} 2 / \mathrm{H} \infty$," Journal of Nanjing University of Aeronautics \& Astronautics, vol. 36, pp. 57-61, 2004.

[18] D. Smallwood, "Minimum input trace for multiple input multiple output linear systems," Journal of the IEST, vol. 56, no. 2, pp. 57-67, 2013.

[19] D. O. Smallwood, "Multiple-input multiple-output (mimo) linear systems extreme inputs/outputs," Shock and Vibration, vol. 14, no. 2, pp. 107-131, 2007. 